Abanico Agroforestal. Enero-Diciembre 2021; 3:1-12. http://dx.doi.org/10.37114/abaagrof/2021.6 Artículo Original. Recibido: 12/04/2021. Aceptado: 27/09/2021. Publicado: 10/10/2021. Clave:2021-22.

\title{
Efecto del ácido linoleico conjugado sobre la calidad de la carne de conejas
}

\author{
Effect of conjugated linoleic acid on quality of rabbit meat
}

\section{Regules-Rivera Hebert ${ }^{11 D}$, González-Montiel Lucio ${ }^{21 D}$, Hernández-Bautista Jorge ${ }^{31 D}$, Ramírez-Ordoñes Sergio ${ }^{4 I D}$, Aguilera-Morales Martha ${ }^{4 I D}$, Antonio-Cisneros Cynthia*41D}

${ }^{1}$ Universidad para el Bienestar Benito Juárez García. San Juan Bautista Cuicatlán Oaxaca. ²Universidad de la Cañada. Teotitlán de Flores Magón, Oaxaca. ${ }^{3}$ Universidad Autónoma Benito Juárez Oaxaca. ${ }^{4}$ Universidad del Papaloapan, campus Tuxtepec y Loma Bonita. Oaxaca. *Autor responsable y de correspondencia: Antonio-Cisneros Cynthia. Circuito central \# 200. Colonia parque industrial, cp. 68301. Tuxtepec, Oaxaca. México. hebert.regulesrivera@gmail.com, luciogonzalez@unpa.edu.mx, jorgebuajo@gmail.com, mvzsramirez@mail.com, aguilena02@hotmail.com, cynthiamagalyac@gmail.com.mx.

\section{RESUMEN}

El uso de ácidos grasos en suplementos para animales de consumo humano es para mejorar la salud del animal y disminuir el uso de medicamentos. El CLA es un compuesto bioactivo y se puede incorporar en la dieta de animales no rumiantes. El objetivo de este experimento fue evaluar el efecto de la incorporación de CLA en la dieta de conejas Nueva Zelanda, sobre la calidad de la carne. Las variables evaluadas fueron la calidad microbiológica de cecótrofos: carga microbiana de BAL, enterobacterias y clostridium spp., los parámetros zootécnicos y la calidad fisicoquímica de la carne de conejas al sacrificio. Los resultados obtenidos mostraron presencia de BAL y Clostridium y ausencia de Enterobacterias durante el tiempo del estudio. El análisis proximal de la carne de conejas no mostró diferencia $(p>0.05)$ en el peso vivo (PV), ganancia de peso (GP), eficiencia alimenticia (EA) y conversión alimenticia (CA) en los tratamientos y el testigo. Pero la CRA y el color mostraron diferencia $(p \leq 0.05)$ entre los músculos LD y BF. El perfil de $A G$ mostró la presencia de CLA en el muslo de las conejas.

Palabras clave: CLA, conejas, CRA, cecótrofos, $\mathrm{pH}$, color.

\begin{abstract}
The use of fatty acids in food animal supplements is to improve animal health and decrease the use of medications. CLA is a bioactive compound and can be incorporated into the diet of non-ruminant animals. The objective of this experiment was to evaluate the effect of incorporating CLA into the diet of New Zealand rabbits on meat quality. The variables evaluated were the microbiological quality of cecotrophs: microbial load of LAB, enterobacteria and clostridium spp., zootechnical parameters and physicochemical quality of meat from rabbits at slaughter. The results obtained showed the presence of $L A B$ and Clostridium and the absence of Enterobacteriaceae during the study period. Proximal analysis of rabbit meat showed no difference $(p>0.05)$ in live weight (BW), weight gain (WG), feed efficiency (FE) and feed conversion ratio $(F C R)$ in the treatments and the control. But CRA and color showed difference $(p \leq 0.05)$ between LD and $\mathrm{BF}$ muscles. The GA profile showed the presence of CLA in the thigh of rabbits.
\end{abstract}

Keywords: CLA, rabbits, CRA, cecotrophs, $\mathrm{pH}$, color. 


\section{INTRODUCCIÓN}

El término CLA, por sus siglas en inglés, describe los isómeros geométricos y posicionales del ácido linoleico C18:2 que contiene dobles enlaces en configuración cis o trans a lo largo de la cadena de 18 carbonos. El resultado de la biohidrogenación microbiana del C18:2 y linolénico C18:3 es la formación de 28 isómeros posibles, donde los isómeros cis-9, trans-11 y trans-10, cis-12 son los más activos en cáncer, obesidad, inflamación, diabetes y aterosclerosis (Castro-Webb et al., 2012; Yang et al., 2015; Jelińska et al., 2017). Los alimentos agropecuarios con CLA son la leche, carne y subproductos derivado de estos; la eficiencia de la producción y la calidad de la carne puede mejorarse al incluir CLA en la dieta de las especies pecuarias. Esta concentración depende de factores ante y posmorten, es decir, raza, clima, salud intestinal, tipo de producción, tipo de músculo y materias primas.

La carne de conejo se considera un alimento funcional (Dalle Zotte \& Szendrő, 2011), porque el consumo regular puede proporcionar compuestos bioactivos: vitaminas y minerales, antioxidantes y AG como el CLA. Los conejos son una especie pequeña con ventajas como las técnicas de crianza, factores biológicos como edad y peso, efectos genéticos y de raza y procesos tecnológicos de transformación de carne que no tienen especies mayores como las aves de corral, cerdos y bovinos. El objetivo de esta investigación fue evaluar el efecto del CLA en la dieta de conejas Nueva Zelanda sobre la calidad de la carne.

\section{MATERIAL Y MÉTODOS}

\section{Condiciones experimentales}

Las conejas Nueva Zelanda $(n=15)$ se adquirieron en la granja cunícola UNAM, México, y trasladadas vía terrestre hasta la posta zootécnica de la Universidad del Papaloapan, campus Loma Bonita, Oaxaca. El estudio se dividió en dos etapas: la etapa con probiótico y la etapa con CLA. Las hembras recién destetadas (30 d) presentaron fecha de nacimiento y peso similar $(762 \pm 50.9 \mathrm{~g})$. La población se dividió en dos grupos experimentales: testigo $(\mathrm{T})$ y tratamiento probiótico $(\mathrm{P})$, alojadas en jaulas dobles, de malla galvanizada cuyas dimensiones fueron $90 \times 60 \times 40 \mathrm{~cm}$. Los dos grupos: T y $P$ fueron alimentados ad libitum con alimento base comercial (Conejina TurboMR, Purina, México) pero al grupo $\mathrm{P}$ se le administró probiótico BioPlus $2 \mathrm{~B}^{\circledR}$ (Chr. Hansen BioSystems $\mathrm{A} / \mathrm{S}$, Denmark), la etapa de probiótico duró seis semanas. Después, en la etapa de CLA (séptima semana), la población fue dividida en tres grupos: testigo $(T)$, tratamiento $1\left(T_{1}\right)$ y tratamiento $2\left(\mathrm{~T}_{2}\right)$, con cinco conejas cada uno. Las hembras fueron distribuidas para controlar ruidos experimentales y que permitieran la coincidencia de características como edad, peso vivo, genotipo, origen (misma granja) y manejo. 


\section{Dietas experimentales}

Los tres grupos se alimentaron con una dieta a base de cereales, minerales y aceites esenciales (Cuadro 1), pero diferente contenido de CLA (Corino et al., 2003). Las dietas se prepararon a mano, sin peletizado ni extrusión.

Cuadro 1. Ingredientes empleados en las dietas para conejas

\begin{tabular}{llll}
\hline Ingrediente, $(\mathrm{g})$ & $\mathrm{T}$ & $\mathrm{T}_{1}$ & $\mathrm{~T}_{2}$ \\
\hline Alfalfa heno & 52 & 52 & 52 \\
Maíz molido & 18 & 18 & 18 \\
Pasta de soya & 12 & 12 & 12 \\
Avena & 9 & 9 & 9 \\
Salvado de trigo & 5 & 5 & 5 \\
Sales y minerales & 1.5 & 1.5 & 1.5 \\
Aceite de canola & 1.5 & 1.1 & 0.7 \\
Metionina sintética & 0.5 & 0.5 & 0.5 \\
CLA & 0 & 0.4 & 0.8
\end{tabular}

$\mathrm{T}=\sin \mathrm{CLA} ; \mathrm{T}_{1}=0.4 \mathrm{~g} \mathrm{CLA} \mathrm{kg}^{-1} ; \mathrm{T}_{2}=0.8 \mathrm{~g} \mathrm{CLA} \mathrm{kg}^{-1}$. Proteína: $20.5 \pm 0.9 \%$, EE: $2.8 \pm 0.1 \%$, Ceniza: $6 \pm 0.1 \%$ y Fibra cruda: $19 \pm 0.8 \%$

El CLA fue adquirido del proveedor $\mathrm{GNC}{ }^{\circledR}$ presentación en frasco de 90 cápsulas de 1.6 g, cada una con $55 \mathrm{mg}$ de CLA. Cada ración contenía CLA administrado en dilución acuosa con $20 \mathrm{~mL}$ de agua destilada. La aplicación fue después de preparar la ración completa y por atomización manual. El tiempo que duró la segunda etapa fue de seis semanas y el tiempo total 12 semanas.

\section{Calidad microbiológica}

Las heces blandas (cecótrofos) fueron analizadas como parte del comportamiento alimentario normal en $\mathrm{T}$ y $\mathrm{P}$. El estudio microbiológico comprendió el conteo de tres grupos microbianos: bacterias acidolácticas (BAL), enterobacterias y clostridium. El procedimiento para la toma, manejo, transporte, preparación y dilución de las muestras se llevó a cabo de acuerdo a Linaje et al., (2004). La identificación de BAL se realizó sobre Agar MRS, las enterobaterias con Agar MacConkey y para clostridium se utilizó Agar Reinforced Clostridial. Los medios de cultivos fueron preparados de acuerdo a las especificaciones del proveedor Fluka Analytical ${ }^{\odot}$, Suiza. Durante la recolección de los cecótrofos se utilizaron pinzas, guantes y tubos de ensayo estériles, fueron trasladados al laboratorio de análisis químico y almacenados en congelación a $-18^{\circ} \mathrm{C}$.

El tratamiento de $1 \mathrm{~g}$ de muestra de cecótrofos se transfirió a bolsas estériles para Stomacher, el agua peptonada estéril al $0.1 \%$ (1 $\mathrm{g}$ de peptona, $8.5 \mathrm{~g}$ de NaCl en $1000 \mathrm{~mL}$ de agua) se adicionó hasta obtener una dilución 1:10, la mezcla se homogenizó durante 
1-2 min. Las alícuotas apropiadas de las diluciones se tomaron de tubos de ensayo con agua peptonada estéril al $0.1 \%$. El análisis se realizó por triplicado y las colonias se contaron después del tiempo de incubación. El criterio utilizado en la selección de las cajas fue de 30 y 300 colonias. Los recuentos obtenidos se expresaron como Log10 de UFC $g^{-1}$.

\section{Parámetros zootécnicos}

El peso vivo (PV) de las conejas se registró por las mañanas semanalmente, durante el tiempo que duró el experimento y antes de suministrar la dieta experimental. La eficiencia alimenticia (EA), se determinó con la expresión 1. En la práctica, el inverso de esta relación es conocida como conversión alimenticia $(C A)$ calculada con la expresión 2 y se usa en la crianza de animales para referirse a la conversión de alimento en ganancia de peso. En todos los casos el peso se obtuvo con una báscula multifuncional Torrey ${ }^{\odot}$, México, modelo LPCR.

\section{$E A=($ aumento de peso diario promedio/alimentación diaria promedio $)$ $C A=(1 / E A)$

Estas expresiones son indicadores de nutrición, la primera mide la eficiencia biológica, mientas que la segunda es una medida económica de alimentación. Los animales con un valor de CA bajo tienden a ser más eficientes, (Gaillard et al., 2020), sin embargo, son diferentes dependiendo de la especie, la genética, estado de salud, prácticas de alimentación y condiciones ambientales. Por otro lado, las conejas fueron insensibilizadas y sacrificadas de acuerdos a la NOM-033-SSA1, en el interior del laboratorio químicobiológico de la universidad. La canal de conejas fue congelada después del sacrificio a $5^{\circ} \mathrm{C}$ hasta su análisis posterior. El peso vivo (PV) se registró antes del sacrificio, el peso de la canal fría (CF) y despojos (D) que corresponden a vísceras, patas, cabeza y cola se obtuvieron después del sacrificio. La ganancia de peso se obtuvo por diferencia mientras que, la grasa perirenal (GPR) e interescapular (GI) se obtuvieron del área de los riñones y la sección vertebral externa respectivamente. Para todas las determinaciones se utilizó una balanza granataria marca Ohaus ${ }^{\odot}$ modelo CS-5000.

\section{Calidad fisicoquímica}

La calidad fisicoquímica se analizó después del sacrificio y a las $24 \mathrm{~h}$ posmorten en canales procedentes de dos músculos diferentes; longissimus dorsis (LD) y bíceps femoris (BF). El análisis proximal materia seca (MS), humedad $(\mathrm{H})$, cenizas $(\mathrm{C})$, extracto etéreo (EE) y proteína bruta (PB) (AOAC, 1990) se llevó a cabo por triplicado. El potencial de hidrógeno $(\mathrm{pH})$ se analizó con un medidor de $\mathrm{pH}$ Orion Star A211, Thermo Scientific de acuerdo a la NOM-317-S-1978. La saturación de color ( ${ }^{*} a,{ }^{*} b$ y *L), se analizó 
utilizando un colorímetro UltraScan® Vis (HunterLab, H unter Associates Laboratory Inc., Hills Road, Reston, Virginia USA). La lectura de color se determinó en ambos músculos, en la zona visible de (400 a $700 \mathrm{~nm}$ ). La capacidad de retención de agua (CRA), se analizó utilizando $\mathrm{NaCl} 0.6 \mathrm{M}$ como solución añadida, la muestra se dejó reposar por 30 min y se centrifugó a $3000 \mathrm{rpm}$ durante $15 \mathrm{~min}$. El sobrenadante se cuantificó como porcentaje de agua retenido en la muestra. El perfil de AG, se cuantificó con una extracción sólido-líquido y una mezcla cloroformo-metanol 2:1 v/v, (Figueiredo et al., 2016). La solución fue analizada por cromatografía de gases (CG) con un cromatógrafo HP equipado con detector de ionización de flama y una columna capilar SupelcoWax-10 de $100 \mathrm{~m} \times 0.25 \mathrm{~mm}$ de diámetro y un espesor de película de $0.2 \mu \mathrm{m}$. Los resultados obtenidos fueron expresados en \% de AG.

\section{Análisis estadístico}

Los datos obtenidos fueron normalizados y analizados mediante un análisis de varianza y una comparación de medias de Tukey con un nivel de significancia $p \leq 0.05$.

\section{Calidad microbiológica}

\section{RESULTADOS}

El recuento promedio de BAL durante el tiempo que duró el experimento fue $8.27+0.72$ UFC $g^{-1}$ y $8.37+0.62$ UFC $g^{-1}$ para $T$ y $P$, respectivamente. La Figura 1 muestra el comportamiento de BAL e indican que existe una diferencia entre los dos grupos.

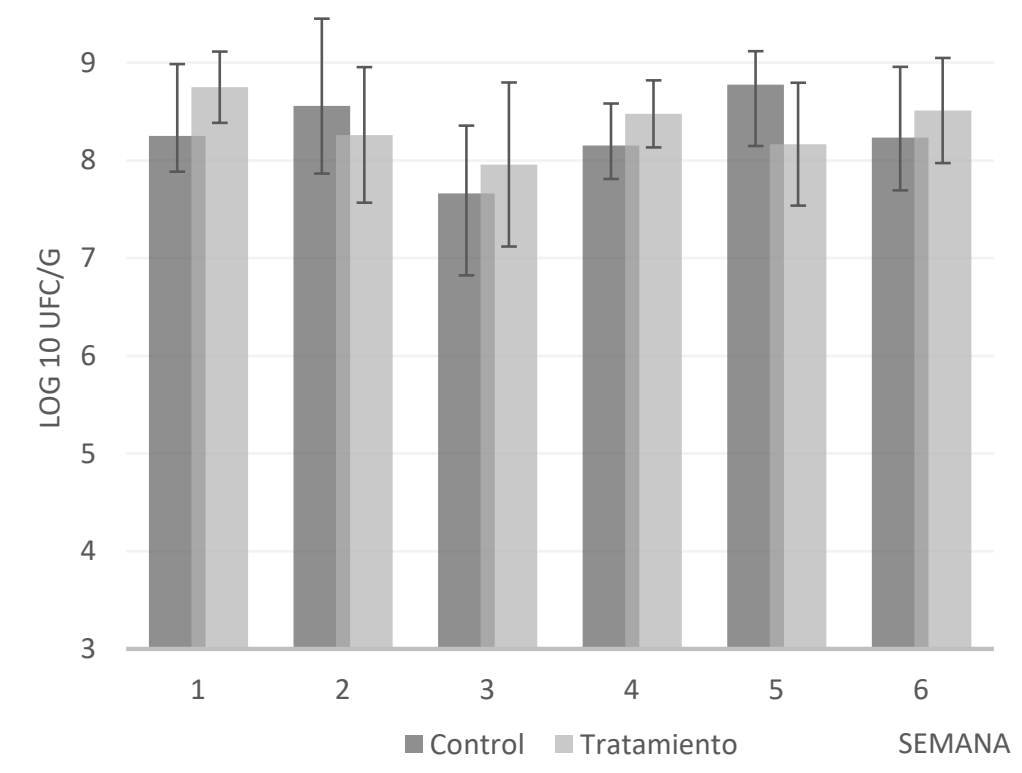

Figura 1. Cuantificación de BAL en cecotrofos de conejas durante 6 semanas 
El promedio de las enterobacterias en T fue $6.64 \pm 1.85 \mathrm{Log}_{10}$ UFC $\mathrm{g}^{-1}$ y para $\mathrm{P}$ no hubo crecimiento de este grupo bacteriano, incluso desde el primer muestreo. Finalmente, el recuento de Clostridium spp., fue de $8.31 \pm 0.87$ UFC g ${ }^{-1}$ y $8.39 \pm 0.60$ UFC g ${ }^{-1}$ para T y $P$, respectivamente.

\section{Parámetros zootécnicos}

EI PV de las conejas es mayor en T comparado con $\mathrm{T}_{1}$ y $\mathrm{T}_{2}$ a pesar de que GP es la misma para todos los grupos (Cuadro 2). El peso de CF se obtiene después del sacrificio y se observó que $T_{1}>T_{>} T_{2}$ y es consistente con $D$ donde se muestra que $T_{1}$ y $T_{2}$ representan una cantidad mayor de despojos (piel, patas y cola), que también pueden ser aprovechados como subproductos (artesanías locales). Las vísceras, sangre y órganos pueden transformarse en harina, ingrediente de alimento para animales. La relación de EA es la misma en $T_{1}$ y $T_{2}$ y $C A$ presenta un valor de 2.2 y 2.1 respectivamente.

Cuadro 2. Parámetros zootécnicos de conejas suplementadas con CLA

\begin{tabular}{lllll}
\hline Variable & $\mathrm{T}$ & $\mathrm{T}_{1}$ & $\mathrm{~T}_{2}$ & \pm \\
\hline $\mathrm{PV}(\mathrm{kg})$ & 2.1 & 2.4 & 2.3 & 1.4 \\
GP, $(\mathrm{kg})$ & 1.1 & 1.1 & 1.1 & 0.01 \\
CF $(\mathrm{kg})$ & 1.2 & 1.3 & 1.0 & 0.05 \\
D $(\mathrm{kg})$ & 0.9 & 1.1 & 1.3 & 0.05 \\
EA & 0.3 & 0.2 & 0.2 & 0.01 \\
CA & 2.0 & 2.2 & 2.1 & 0.03 \\
GPR (g) & 4.8 & 8.8 & 9.5 & 2.5 \\
GI (g) & 1.0 & 2.8 & 2.2 & 0.9
\end{tabular}

$\mathrm{T}=\sin \mathrm{CLA}, \mathrm{T}_{1}=0.4 \mathrm{~g}$ CLA kg${ }^{-1}, \mathrm{~T}_{2}=0.8 \mathrm{~g}$ CLA kg${ }^{-1}$. PV=peso vivo, CF=canal fría, $\mathrm{D}=$ despojos, $\mathrm{CA}=$ conversión alimenticia, $\mathrm{EA}=$ eficiencia alimenticia, $\mathrm{GP}=$ ganancia de peso, GPR=grasa perirrenal, $\mathrm{Gl}=$ grasa interescapular, $\mathrm{DE}=$ desviación estándar.

Finalmente, los valores de GPR son diferentes tanto en los tratamientos como en T y este comportamiento se presenta en los valores de Gl.

\section{Calidad fisicoquímica}

La composición química proximal se muestra en el Cuadro 3, el contenido de $\mathrm{H}$ es de 74$73 \%$, similar a lo reportado por la FAO, el cual reporta $74.9 \%$ en carne de conejo. La MS, por tanto, presentó el contenido más alto del análisis proximal (25-26\%). El contenido de $\mathrm{C}$ representa los minerales, es decir, los iones esenciales para el humano; éste fue diferente únicamente cuando se comparó por tratamiento (Cuadro 3). 
Cuadro 3. Composición proximal en carne de coneja suplementadas con CLA

\begin{tabular}{llllllllll}
\hline$\%$ & $\mathrm{~T}$ & $\mathrm{~T}_{1}$ & $\mathrm{~T}_{2}$ & $\mathrm{EEM}$ & Prob & LD & $\mathrm{BF}$ & EEM & Prob \\
\hline $\mathrm{MS}$ & 25.66 & 26.33 & 25.84 & 0.410 & $\mathrm{NS}$ & 25.94 & 25.95 & 0.335 & $\mathrm{NS}$ \\
$\mathrm{P}$ & 22.44 & 23.07 & 23.06 & 0.396 & $\mathrm{NS}$ & 22.51 & 22.52 & 0.373 & $\mathrm{NS}$ \\
$\mathrm{C}$ & $1.37 \mathrm{ab}$ & $1.29 \mathrm{a}$ & $1.47 \mathrm{~b}$ & 0.038 & 0.005 & 1.38 & 1.39 & 0.031 & $\mathrm{NS}$ \\
$\mathrm{EE}$ & 1.27 & 1.30 & 1.17 & 0.208 & $\mathrm{NS}$ & 1.23 & 1.24 & 0.170 & $\mathrm{NS}$
\end{tabular}

Testigo =sin CLA, $\mathrm{T}_{1}=0.4 \mathrm{gCLA} \mathrm{Kg}^{-1}, \mathrm{~T}_{2}=0.8 \mathrm{gCLA} \mathrm{Kg}^{-1}$. LD= Longissimus dorsis, BF= Biceps femoris. Los valores presentan el promedio \pm SEM. abcd, letras diferentes en cada columna indican diferencia significativa $(\mathrm{p} \leq 0.05)$. EEM=error estándar de la media, $N S=$ no significativo.

Los parámetros de calidad tecnológica de la carne se muestran en el Cuadro 4, la CRA cambia durante el tiempo posmorten y baja a medida que se llega a la maduración de la carne. Se observó que existe diferencia estadística entre tratamientos $\left(\mathrm{T}_{1}\right.$ y $\left.\mathrm{T}_{2}\right)$ y músculos (LD y BF) después de 1 y $24 \mathrm{~h}$ de sacrificio. La CRA es un parámetro que depende del pH el cual provoca la pérdida de humedad, el cambio de textura, el aumento de la terneza y la diferencia de color. El cambio de $\mathrm{pH}$ hacia valores ácidos, está asociado al proceso irreversible de contracción muscular a nivel de las cadenas de actina-miosina y por la producción de ácido láctico que disminuye el $\mathrm{pH}$ hasta 5.4. El valor de $\mathrm{pH}$ en este estudio, muestra cambios significativos solo entre tratamientos ( $\mathrm{T}_{1}$ y $\mathrm{T}_{2}$ ) después de 1 y $24 \mathrm{~h}$ de sacrificio. Los factores asociados con los cambios en CRA y pH son la producción de ácido láctico y por lo tanto la disminución de $\mathrm{pH}$, el consumo de glucógeno en el músculo, la disminución de compuestos energéticos (ADP y ATP) que inician la rigidez cadavérica y, por lo tanto, la desnaturalización de proteínas.

El color está asociado con el estado físico $\left(b^{*}\right)$, químico $\left(a^{*}\right)$ y el contenido de hemoglobina $\left(L^{*}\right)$. En todos los casos el parámetro $L>b>a$ (Cuadro 4 ). El parámetro $L^{*}$ asociado con el contenido de hemoglobina. fue el único parámetro que no presentó diferencia significativa cuando se analizaron los resultados por tratamientos ( $\mathrm{T}_{1}$ y $\left.\mathrm{T}_{2}\right)$ y por tipo de músculo (LD y BF). Lo anterior es importante debido a que el color no se ve afectado por las dietas probadas y tampoco cuando este análisis se realiza en dos músculos comerciales diferentes. El parámetro $b^{*}$ fue diferente estadísticamente cuando fue comparado por tratamientos $\left(\mathrm{T}_{1}\right.$ y $\left.\mathrm{T}_{2}\right)$ mientras que $\mathrm{a}^{*}$ no fue diferente cuando se evaluaron los resultados en ambas condiciones. 
Cuadro 4. Parámetros de calidad tecnológica de la carne conejas suplementadas con CLA

\begin{tabular}{|c|c|c|c|c|c|c|c|c|c|}
\hline$\%$ & $T$ & $\mathrm{~T}_{1}$ & $\mathrm{~T}_{2}$ & EEM & Prob & LD & $\mathrm{BF}$ & EEM & Prob \\
\hline $\mathrm{CRA}_{1 \mathrm{~h}}$ & 18.65ab & $19.08 \mathrm{~b}$ & $17.53 a$ & 0.501 & 0.04 & 17.79 & 19.04 & 0.409 & 0.05 \\
\hline $\mathrm{CRA}_{24 \mathrm{~h}}$ & $9.36 b$ & $8.88 a$ & $8.86 a$ & 0.150 & 0.04 & 8.43 & 9.64 & 0.123 & 0.01 \\
\hline $\mathrm{pH}_{1 \mathrm{~h}}$ & 6.42 & 6.42 & 6.52 & 0.050 & NS & 6.84 & 6.07 & 0.041 & 0.01 \\
\hline $\mathrm{pH}_{24 \mathrm{~h}}$ & 5.94 & 5.98 & 5.96 & 0.065 & NS & 6.29 & 5.66 & 0.053 & 0.01 \\
\hline \multicolumn{10}{|l|}{ Color24h } \\
\hline$L^{*}$ & 62.44 & 60.36 & 62.19 & 0.976 & NS & 60.96 & 62.36 & 0.797 & NS \\
\hline$b^{*}$ & 12.30 & 12.37 & 11.86 & 0.664 & NS & 10.31 & 14.04 & 0.542 & 0.01 \\
\hline$a^{*}$ & $-0.05 a$ & $-0.28 a b$ & $-0.57 b$ & 0.176 & 0.05 & -2.82 & 2.22 & 0.144 & 0.01 \\
\hline
\end{tabular}

Testigo $=\sin \mathrm{CLA}, \mathrm{T}_{1}=0.4 \mathrm{gCLA} \mathrm{Kg}^{-1}, \mathrm{~T}_{2}=0.8 \mathrm{gCLA} \mathrm{Kg}^{-1}$. $\mathrm{LD}=$ Longissimus dorsis, $\mathrm{BF}=$ Biceps femoris. $C R A=$ capacidad de retención de agua, $L^{*}=$ luminosidad, $a^{*}=$ rojo, $b^{*}=$ amarillo. Los valores presentan el promedio $\pm S E M$. abcd, letras diferentes en cada columna indican diferencia significativa $(p \leq 0.05)$. NS $=$ no significativo.

\section{Perfil de ácidos grasos}

Por otro lado, el perfil de AG se muestra en el Cuadro 5, se observa que la composición en la carne de coneja procedente del músculo LD fue 18:1>C14:0>C16:0>AGPI, presentando diferencia significativa únicamente en el AG C18:1 entre los tratamientos probados. Mientras que el músculo BF la composición fue C18:2>C18:1>C16:0>C18:2, $\mathrm{Cot}_{11}>\mathrm{C} 18: 2, \mathrm{C}_{12} \mathrm{t}_{10}>\mathrm{C} 14: 0$.

Cuadro 5. Perfil de AG en carne de conejas suplementadas con CLA

\begin{tabular}{llllllll}
\hline & \multicolumn{3}{c}{ Longissimus dorsis } & \multicolumn{5}{c}{ Biceps femoris } \\
& $\mathrm{T}$ & $\mathrm{T}_{1}$ & $\mathrm{~T}_{2}$ & $\mathrm{~T}$ & $\mathrm{~T}_{1}$ & $\mathrm{~T}_{2}$ & $\mathrm{EEM}$ \\
\hline $\mathrm{C} 14: 0$ & $21.4 \mathrm{~b}$ & $23.91 \mathrm{c}$ & $23.50 \mathrm{c}$ & $\mathrm{sd}$ & $\mathrm{sd}$ & $\mathrm{sd}$ & 0.55 \\
$\mathrm{C} 16: 0$ & $8.49 \mathrm{a}$ & $9.12 \mathrm{a}$ & $8.73 \mathrm{a}$ & $11.38 \mathrm{~b}$ & $21.79 \mathrm{~d}$ & $20.10 \mathrm{c}$ & 0.41 \\
$\mathrm{C} 18: 1$ & $27.16 \mathrm{de}$ & $24.40 \mathrm{bc}$ & $28.13 \mathrm{e}$ & $13.92 \mathrm{a}$ & $25.78 \mathrm{~cd}$ & $24.08 \mathrm{~b}$ & 0.52 \\
$\mathrm{C} 18: 2$ & $\mathrm{sd}$ & $\mathrm{sd}$ & $\mathrm{sd}$ & $16.51 \mathrm{~b}$ & $25.84 \mathrm{c}$ & $26.94 \mathrm{c}$ & 0.62 \\
$\mathrm{C} 18: 2, \mathrm{c} 9111$ & $\mathrm{sd}$ & $\mathrm{sd}$ & $\mathrm{sd}$ & $\mathrm{sd}$ & $0.487 \mathrm{~b}$ & $0.710 \mathrm{c}$ & 0.03 \\
C18:2, c12t10 & sd & $\mathrm{sd}$ & $\mathrm{sd}$ & $5.3 \mathrm{~b}$ & $\mathrm{sd}$ & $\mathrm{sd}$ & 0.06
\end{tabular}

Testigo $=\sin$ CLA, $\mathrm{T}_{1}=0.4 \mathrm{gCLA} \mathrm{kg}^{-1}, \mathrm{~T}_{2}=0.8 \mathrm{gCLA} \mathrm{kg}^{-1}$. Los valores presentan el promedio $\pm \mathrm{SEM}, \mathrm{ab} c \mathrm{~d}$, letras diferentes en cada columna indican diferencia significativa $(p \leq 0.05)$, $s d=s i n$ detectar.

\section{DISCUSIÓN}

Latorre et al., (2014) evaluaron la persistencia de esporas de Bacillus subtilis, un tipo de $\mathrm{BAL}$, a través del tracto gastrointestinal (TGI), los resultados mostraron que aproximadamente el $90 \%$ de las esporas germinan en el TGl. Los cambios relacionados con las diferentes condiciones fisiológicas, nutricionales y microbiológicas pueden ser 
comparadas con BAL, éstas tienen la capacidad para germinar a nivel TGl y sobrevivir en el ambiente probado, tal como se aprecia en la Figura 1. El incremento de las BAL en este estudio, durante las semanas 4 y 6 permite inferir en un posible aumento de la producción de sustancias antimicrobianas que ayudan a la salud del TGI del conejo. La administración de probiótico cumple la función de prevenir o retardar la aparición de enfermedades gastrointestinales y los conejos que consumieron probiótico en la dieta mostraron condición de salud adecuadas (Gaggia et al., 2010). La bacteria E. coli pertenece a la familia de enterobacterias y su presencia está asociada a la diarrea en animales recién destetados y la sustitución de los antibióticos en la dieta. Las características zootécnicas pueden mejorar significativamente debido al desarrollo y el mantenimiento de la microbiota cecal que promueve el estado de salud de los animales. Los probióticos Saccharomyces cerevisiae y Enterococcus faecilum fueron administrados oralmente durante dos semanas en conejos sanos; el tratamiento no afectó los niveles fecales de especies de bacteroides, fibrobacter succinogenes o clostridium spiroforme, (Summa \& Brandao, 2017).

Los parámetros de calidad tecnológica de las carnes, pH, CRA y color demostraron cambios esperados mientras que los parámetros zootécnicos fueron bajos a los reportados por Palma \& Hurtado (2009), ellos alimentaron gazapos machos durante $60 \mathrm{~d}$ con una dieta combinada de alimento comercial $(A C)$ y mango criollo fresco (MC), la CA fue de 2.41 a 2.13 en dietas con 60 y $40 \mathrm{~g}$ de AC y 60 y $80 \mathrm{~g}$ de MC. En otro estudio, (Corino et al., 2003) encontraron que los animales suplementados con CLA no obtuvieron diferencias significativas en los parámetros productivos. Pérez-Martínez et. al., (2018) evaluaron parámetros productivos en conejos alimentados con diferentes partes de la planta Tithonia tubaeformis, ellos no encontraron diferencia significativa en las variables medidas, sin embargo, la CA fue de 3.18 a 3.41. dichos resultados son similares a lo reportado por Meineri et al., (2010) y Dalle Zotte \& Szendro (2011).

Malavé (2013), reportó que la suplementación alimenticia de diferentes animales no afecto significativamente la composición química proximal, resultados consistentes con este estudio donde se observó diferencia únicamente en el contenido de T (1.47-1.29\%). Estos resultados indican la presencia de CLA, cis-9, trans-11, en la carne procedente del músculo $\mathrm{BF}$ en ambos tratamientos y es mayor en $\mathrm{T}_{2}$, es decir, cuando las conejas son alimentadas con $0.8 \mathrm{gCLA} \mathrm{Kg}^{-1}$. Corino et al., (2003) determinaron el perfil de AG en Longissimus lumborum en conejos suplementados con $0.5 \%$ CLA y obtuvieron $20 \%$ de C18:1, 29\% de C18:2n6 y 25\% de C16:0. En otro estudio, se reportó la proporción relativa de diferentes tipos de $A G$ en $L D$, alcanzando $38 \%$ de $A G$ saturados, $28 \%$ de AG monoinsaturados y $32 \%$ de AG poli-insaturados. Estas proporciones de AG permiten comparar la carne de conejo con otras especies como el cerdo, pollo, ternero y res (Dalle Zotte \& Szendrő, 2011). Sin embargo, la carne de conejo tiene características funcionales 
como bajo contenido de colesterol, alto contenido de proteína y aminoácidos esenciales; especialmente en LD, digestibilidad alta y niveles altos de AG poli-insaturados que permite considerarlo como un alimento funcional con propiedades nutricionales y dietéticas (Dalle \& Szendrö, 2011).

\section{CONCLUSIONES}

La calidad de la carne depende de varios parámetros, en este estudio se analizó la dieta enriquecida con CLA sobre la calidad microbiológica, productiva y fisicoquímica. La calidad microbiológica indicó que las BAL están presentes durante el tiempo que duro el estudio (seis semanas) y es suficiente para inhibir a las enterobacterias. comportamiento es importante considerando que en las primeras semanas de vida se adquiere inmunidad ante la presencia de diversos patógenos que dañan la salud del animal. El peso comercial se alcanzó en 80 d de alimentación con una CA baja y un rendimiento de la canal de acuerdo a lo esperado. La calidad productiva no se afecta cuando se suministran dos dietas diferentes, sin embargo, la evaluación de la calidad fisicoquímica de la carne, indicó que la CRA es el parámetro afectado en la evaluación y es reflejado en el estado fisicoquímico de la hemoglobina. El perfil de AG demostró que los AGS no cambian por el tipo de músculo y se demostró la presencia de CLA al menos en el músculo BF. Lo anterior puede emplearse para considerar la carne de BF o muslo, una fuente alternativa de CLA.

\section{AGRADECIMIENTOS}

Este estudio fue financiado por PRODEP con número de registro PROMEP/103.5/13/7556. Se agradece al Instituto Tecnológico de México, campus Tuxtepec por las facilidades proporcionadas en la realización del análisis cromatográfico. El estudio de la calidad microbiológica se realizó en la Universidad de la Cañada y el estudio de calidad productiva y fisicoquímica en la Universidad del Papaloapan, campus Loma Bonita. Se agradece por su colaboración a Javier Vázquez Roble y José Espinoza Azamar.

\section{LITERATURA CITADA}

AOAC (Association of Official Analytical Chemists). 1990. Official Methods of Analysis. International $17^{\text {th }}$ edition. Gaitherbusrg, MD. USA. Pp 69-88. ISBN:0-935584-42-0. https://law.resource.org/pub/us/cfr/ibr/002/aoac.methods.1.1990.pdf

CASTRO-WEBB N, Ruiz-Narvaez E, Campos H. 2012. Cross-sectional study of conjugated linoleic acid in adipose tissue and risk of diabetes. American Journal of Clinical Nutrition. 96:175-181. https://doi.org/10.3945 / ajcn.111.011858 
CORINO C, Filetti F, Gambacorta M, Manchisi A, Magni S, Pastorelli G, Rossi R, Maiorano G. 2003. Influence of dietary conjugated linoleic acids (CLA) and age at slaughtering on meat quality and intramuscular collagen in rabbits. Meat Science. 66:97103. https://doi.org/10.1016/S0309-1740(03)00024-X

DALLE ZOTTE A, Szendrö Z. 2011. The role of rabbit meat as functional food. Meat Science. 88: 319-331. https://doi.org/10.1016 / j.meatsci.2011.02.017

FIGUEIREDO I, Claus T, Oliveira Santos Júnior O, Almeida V, Magon T, Visentainer J. 2016. Fast derivatization of fatty acids in different meat samples fos gas chromatography analysis. Journal of Chromatography. 1456: 235-241. https://doi.org/10.1016/j.chroma.2016.06.012

GAGGIA F, Mattarelli P, Biavati B. 2010. Probiotics and prebiotics in animal feeding for safe food production. International Journal of Food Microbiology. 141: S15-S28. https://doi.org/10.1016/j.jifoodmicro.2010.02.031

GAILLARD Ch, Brossard L, Dourmad JY. 2020. Improvement of feed and nutrient efficiency in pig production through precision feeding. Animal Feed Science and Technology. 268:114611. https://doi.org/10.1016/j.anifeedsci.2020.114611

JELIŃSKA M, Bialek A, Gielecińska I, Mojska H, Tokarz A. 2017. Impact of conjugated linoleic acid administered to rats prior and after carcinogenic agent on arachidonic and linoleic acid metabolites in serum and tumors. Prostaglandins, Leukotrienes and Essential Fatty Acids. 126:1-8. https://doi.org/10.1016/j.plefa.2017.08.013

LATORRE JD, Hernández-Velascos X, Kallapura G, Menconi A, Pumford NR, Morgan MJ, Layton SL, Bielke LR, Hargis BM Tellez G. 2014. Evaluation of germination, distribution and persistence of Bacillus subtilis spores through the gastrointestinal tract of chickens. Poultry Science Association Inc. 93:1793-1800. https://doi.org/10.3382/ps.2013-03809

LINAJE R, Coloma MD, Pérez MG Zúñiga M. 2004. Characterization of feacal enterococci from rabbits for the selection of probiotics strains. Journal of Applied Microbiology. 96:761771. https://doi.org/10.1111/j.1365-2672.2004.02191.x

MEINERI G, Cornale P, Tassone S, Peiretti P. 2010. Effects of Chia (Salvia hispanica L.) seed supplementation on rabbit meat quality, oxidative stability and sensory traits. Italian Journal of Animal Science. 9:e10. https://doi.org/10.4081/ijas.2010.e10 
MALAVÉ A, Córdoba L, García R, Méndez N. 2013. Composición bromatológica de la carne de conejos suplementados con mata ratón y cachaza de palma aceitera. Rev. MVZ Córdova. $\quad$ 18:3452-3458. http://www.scielo.org.co/scielo.php?pid=S012202682013000200004\&script=sci_abstract\&tIng=es

PALMA OR, Hurtado EA. 2009. Comportamiento productivo de conejos durante el periodo de crecimiento-engorde alimentados con frutos de mango (Mangifera indica) en sustitución parcial del alimento balanceado comercial. Revista UDO Agrícola. 4:968-971. http://dx.doi.org/10.4067/S0718-34292010000100005

PÉREZ-MARTíNEZ K, García-Valencia S, Soto-Simental S, Zepeda-Bastida A, AyalaMartínez M. 2018. Parámetros productivos de conejos alimentados con diferentes partes de la planta Tithonia tubaeformis. Abanico veterinario. 8:108-114. https://doi.org/10.21929/abavet2018.82.10

SUMMA N, Brandão J. 2017. Evidence-based advances in rabbit medicine. Veterinary clinics of North America: exotic animal practice. 20: 749-771. https://doi.org/10.1016/j.cvex.2017.04.008

YANG B, Chen H, Stanton C, Ross RP, Zhang H, Chen YQ, Chen W. 2015. Review of the roles of conjugated linoleic acid in health and disease. Journal of Functional Foods. 15:314- 325. https://doi.org/10.1016/j.jff.2015.03.050 